\title{
Study on Database Programming and Its Statistics
}

\author{
Wenting $\mathrm{Yu}^{1, \mathrm{a}}$ \\ ${ }^{1}$ University of California, San Diego, La Jolla, CA 92037 \\ ${ }^{a}$ email
}

Keywords: Database Programming, Statistics, Computer Technology

\begin{abstract}
Database programming refers to the database side to complete the application business logic, database monitoring as well as their implementation and use of triggers, stored procedures and stored functions. The main triggers are used to achieve the strengthening of table constraint, table synchronization, and audit functions. Both stored procedures and functions can be implemented by business logic related data, but the only difference between the two is that the stored function must have a return value, whereas the stored procedure does not. The process is referred to below those three collectively. The operation of database can be realized on the application side, as well as be achieved by the process on the database side. However, from the perspective of system performance, the database side can achieve superior implementations to the application-side.
\end{abstract}

\section{Introduction}

Database management system to the application designer provides two interfaces, used to define and manipulate data in the database, thereby enhancing the ability to develop applications. The first is a pre-compiled interface for application developers, which is the most effective method using precompiled interfaces, such that developers can use high-level language and high-level language embedded SQL statements to write applications that use the database. SQL statements to complete the retrieval of data from the database, insert, modify, and delete the main language used manipulate data in memory, the various calculations and logic. Pre-compiler will embed the main language in SQL statements translating into high-level language source code, and then use high-level language compiler to compile it so that run-time libraries are connected to form an executable program.

ProC pre-compiler compiler is embedded in one prematch compiler SQL statements in C language, since the main language is $\mathrm{C}$ language, so most of its programming specifications follow the $\mathrm{C}$ programming language specification. Here refers to the specification database programming language $\mathrm{C}$ embedded SQL statement, then the programming specification, mainly, and database-related part. The second is a database call interface, which allows high-level language program in the form of function calls to directly access data in the database, the database development directly follows high-level language development of norms, just call the database system can function or procedure.

\section{Editing and Variable Naming}

It must be in plain text editing software editing software .2) SQL statements that access a database table names, column names must be in capital, and the main difference in the language in which CB_PASSWORD field names, COMMON_DATA is to show, in capital in the main language and the variable phase difference, of course, did not use the lowercase syntax errors .3) host variables, stored procedures are named the host language and style consistent .4) SQL statements should be used and host language (such as $\mathrm{C} / \mathrm{C}++$, etc.) the same indentation style, that is, in the layout style, the SQL statements and host programming language consistent with recommendation indent of each clause and a keyword EXEC SQL after alignment .5) recommended that the SQL statements in the child new sentence at the division line, in order to make the program easy to read .6) If the SQL clause is too long (> 80 characters), you should write branch, long expression to divide in a new line of low-priority operators at designated a new line to be properly indented, making the layout 
neat, the statement read. indented manner recommended in two ways, one is the host language and consistent one is the alignment operator keyword. examples of the latter are effectively host language use and consistent indentation .7) SQL statements if there are nested statements, nested statements need to be divided and a new line, the new line should be indented to operate word-aligned nested statements .8) SQL language definition stored procedures, triggers, and so must have detailed notes, including features, applications, and which functions or procedures and precautions to use it when accessing instructions .9) defined host variables must be included in EXEC SQLBEGIN DE-CLARE SECTION and EXEC SQL END dECLARE SECTION statement indicating and host variables declared best separated, and separated by a blank line .10) defined SQL stored procedures, triggers, etc. must be included in the EX-EC sQLCREATE and the END-EXEC Room, CREATE statement must be the top grid and END_EXEC writing content FUNCTION keywords such cause must start a new line, and properly indented .11) calls the SQL statements defined in stored procedures, triggers, etc. and must be included in EXEC SQLEXECUTE between END_EXEC statement, EXECUTE statement and END-EXEC statement should be aligned and the current host statements .12) procedures used to prepare indentation style, indent with spaces rather than the TAB key 13) relatively independent blocks and illustrated by variable between blank lines separate 14) an effective amount of at least 20\% Note 15) in the header of the response file Note 16) in response to the function header function Note 17) for important variables, types, content to Note 18) Notes to articulate, precise meaning, to prevent ambiguity; avoid the use of abbreviations in the notes, in particular, is very abbreviations used when the program is changed, Note to update.

\section{Effect of Different Methods to Achieve Database Programming}

In today's web information system oracle + java system has gradually become the mainstream of development, architecture mainly uses the browser + web server + database server Bls architecture.

In the popular jsp + javabean development of web information system, for example, from four aspects to explain, as much as possible with the process to achieve the operation of the database, the operation of the database, the database logic related to the industry as much as possible into the database side to complete.

Our definition of operating system database response time $\mathrm{T}$ is $\mathrm{T}=\mathrm{ttl}+\mathrm{te}+\mathrm{te}+\mathrm{ttZ}$, where there is a web server ttl time to transfer the database server, ttZ is the reverse transmission time, tc is compiled web server code segment time te database operation statement is executed in the database server. Since the page between the browser and web server request and return time has no effect on the research questions, and therefore not be considered.

(1) implement javaBean, with Bean to achieve operation of the database, that is to add a method to execute SQL statements in Bean and add the class attribute to be used to pass parameters or return values are stored. The method (2) in jsp with usebean command, the introduction of the implemented and calls Bean Bean perform operations retrieve data. (3) Remove the Bean attribute values, unified display, generate reports. The implementation process: the page to submit a request to the web server, connect to the database server to run the program and pass the first query to the database, the database each receive a SQL statement to view the store there the same statement, it does not compile, execute and return a result, there is executed, returns the result. Since there are three sQL the following statement, the process is repeated three times, in the worst case, the T (sum) $=4(\mathrm{ttl}+\mathrm{ttz}+\mathrm{te}+\mathrm{te})$, where we assume that similar various time periods of each statement .

(1) to write stored procedures in the database side, and compiled so that the effective .4 statement in this process; (2) in the application, we also have to realize Bean, where you can add a method to create a connection, call stored procedure ; method (3) in jsp with usebean command, the introduction of the implemented aean Bean and calls for the implementation of the operation and to remove the data; (4) the value of property acquired by the jsp page and displayed. Response procedures and time $\mathrm{T}$ : page to submit applications, web server executes the program to create a connection and transfer the call statement, the database execute a stored procedure that returns data. Database process, whether it is a trigger or stored procedures and functions, once compiled correctly, 
it is active, pre-compiled form in the database, and if implemented on the presence of memory, eliminating the need to compile time, so execution speed is very fast. From the above $\mathrm{T}=\mathrm{ttl}+\mathrm{ttZ}+$ 4te.

A transaction is the basic unit of work database, with consistency and isolation characteristics, operation of a thing contains either all executed or all not executed. In fact, the transaction as a whole set of SQL statements, all statements execute successfully the transaction is executed successfully, if there is a not successfully executed, the transaction is rolled back. Database transaction control is an important part of the database, the database version with increased processing power of the database itself is also getting stronger. Of course, the application side have transaction control statements, JDBC in Conneetion class has, rollbaek (), eommit () method, used for transaction control. For example: In a transaction that we have such an operation, to achieve the transfer of archive records in the table. The first table bg_hyssy transfer records to the table bg_hyssyls, and then in the qualifying table bg_hyssq transfer records to the table bg_hyssy, for this to take bg_hyssy empty table, and finally the bg_hyssq emptied in preparation for future work.

Create bg_Hysjlzyis

begin

insert into bg_hy ssyls select* frombg_hyssy;

delete from bg_hyssy:

insert into bg - hyssy (MC, RQ, KSsJ, JSSJ, JE, SQZ, SQRQ,

TBR, DDH)

Select MC, RQ, KSSJ, JSSJ, JE, SQZ, SQRQZ TBR, DDH

From bg_hyssq

Where zthz = 'y';

delete frombg - hyssq;

coMMIt;

EXEPTION

ORTHOR: rollback;

end;

\section{Safety Program Development and Its Application}

ORACLE database has a very strong and very complex security mechanisms, which are commonly used are assigned to users and groups, in general principle is to assign permissions to the database user privileges granted to a minimum, decentralization of authority, the less the more secure. On the application side to realize the operation of the data, which means to give the application to add, delete, change permissions to the database. Conversely, if the database side with the process of realization, just give permission to the application program execution. To add, delete, change operations are put to the database to complete, the application user does not need to have these rights, simply to grant permissions to execute it, thereby reducing the threat to the data.

High-level programming language and the database language, the entire system development is divided into two, a part of staff development database, responsible for the database management system objects, create tables, views, triggers, procedures and functions, etc., to stay out of the application interface. Another part of the development application system, is responsible for a variety of application logic functions are implemented and users of the exchange. Both parties use their own familiar language to work, it will undoubtedly speed up the progress of the project. The operation of the database points to the database server, encapsulate only presented to the application developer interfaces, whether it is from the previous modular programming or from the now popular object-oriented programming is concerned, it is very suitable. Such as: library management program to borrow books, just tell the application database, who would like to take the books, as the stored procedure to determine whether the library card is available, the stock minus a book to borrow flag can not borrow, and so the user It is not known. Just to know whether the loan is successful, and if not, why. It is consistent with the principles of object-oriented programming the black box. This greatly simplifies the application programming end, make the program easier to 
read, but also simplifies the jsp page.

Design applications, logically related activities should be brought together in a single transaction. A well-designed transaction shall include all the steps necessary for a given task, neither more nor less. When updating a table when data, other data in the table must be associated with it at the same time update to ensure data integrity can be achieved in the application of a simple case; if such associated or more frequent updating operation, the trigger should be adopted the data is updated in a transaction should be completed. Expressly requires the level of the transaction, if the transaction higher, to commit statements to determine whether the implementation correctly found commit statement is executed incorrectly, to be prompted or recording. If the transaction less demanding, not to determine whether to commit the statement executed correctly, because the execution is incorrect there is no solution.

\section{Conclusion}

The above are just a few simple examples cited, the process of database, there are three types of triggers, stored procedures and stored functions, they can achieve more powerful database operations function is not limited to the author mentioned above, triggers can be achieved in addition to the application the program can be implemented CRUD operations, mainly used to strengthen the table constraints, remote table synchronization, implements the audit capabilities. Stored procedures and functions, not very different, but the function must return a value. They can do the work of a very strong, basically, all operations on the database can reduce the execution time $t$, eliminating the need for complex transaction control process reduces the amount of information transmission, reducing the number of connections to the database. Of course, everything has advantages and disadvantages, the process using the database will undoubtedly improve application performance, because the presence of memory, it also takes up a lot of system resources, but today, the price of the storage system has been quite low for large applications, it is worth the sacrifice of these resources is also necessary.

\section{References}

[1] H. E. Mic Nay. Enterprise Content Management, an Overview. In Proc. IEEE IntProfessional Communication Conf, (IPCC2002), 2002, 396.402.

[2] James Robertson. How to evaluate a content management ystem[DB/OL]. OnlineArticle, 2005.

[3] Micheal Sokic. Web Content Management System Based on XML NativeDatabase. Croatian Telecom, Jurisiceva 13, Zagreb. HR-I0000, CROATLA, 2004.

[4] Joel Summers, Paul Barford, Nick Dufield, Amos Ron.Ira proving Accuracy inEnd-to-End Packet Loss Measurement. In Proc. ACM SIGCOMM 2005,Philadelphia,USA. August, 2005.

[5] McAvoy T. J. Contemplative stance for chemical process control. Automatic2002, 28(2) :441-442.

[6] Gerald S0M. Distributed Field bus and Control Network. Computer and ControlEngineering Journal 2007, (3):22. 24.

[7] A . Field man, A . C. Gilbert and W. Willinger Data networks as cascades:Investigating them ultifractal nature Of Internet WAN traffic (ComputerCommunication Reviews Vol. 28,No. 4, 2008. 